OPEN ACCESS

Edited by:

Yuli Huang,

Southern Medical University, China

Reviewed by:

Zuheng Liu,

First Affiliated Hospital of Xiamen

University, China

Jin-Jer Chen,

National Taiwan University, Taiwan

${ }^{*}$ Correspondence:

Pingan Chen

cpadejxyx@gzhmu.edu.cn

tThese authors have contributed equally to this work

Specialty section:

This article was submitted to General Cardiovascular Medicine,

a section of the journal

Frontiers in Cardiovascular Medicine

Received: 27 August 2021 Accepted: 08 November 2021 Published: 02 December 2021

Citation:

Chen Y, Guo Z, Li S, Liu Z and Chen P (2021) Spermidine Affects Cardiac Function in Heart Failure Mice by Influencing the Gut Microbiota and Cardiac Galectin-3. Front. Cardiovasc. Med. 8:765591. doi: 10.3389/fcvm.2021.765591

\section{Spermidine Affects Cardiac Function in Heart Failure Mice by Influencing the Gut Microbiota and Cardiac Galectin-3}

\author{
Yufeng Chen ${ }^{1 \dagger}$, Zhiqin Guo ${ }^{1 \dagger}$, Shaonan $\mathrm{Li}^{1,2}$, Zhen Liu ${ }^{1,2}$ and Pingan Chen ${ }^{1 *}$ \\ ${ }^{1}$ Department of Cardiology, The Second Affiliated Hospital, School of Medicine, South China University of Technology, \\ Guangzhou, China, ${ }^{2}$ Department of Cardiology, Guangzhou First People's Hospital, Guangzhou, China
}

Spermidine, which can be synthesized by the gut microbiota, can prevent cardiac hypertrophy and delay the progression to heart failure (HF). However, it is not clear whether the effect of spermidine on cardiac function is mediated by modulating the gut microbiota when HF occurs. Female HF Kunming mice induced by transverse aortic constriction were administered spermidine (HF+S group) or its antagonist ( $\mathrm{HF}+\mathrm{SR}$ group). Echocardiography, messenger ribonucleic acid (RNA) and protein expression of galectin-3 in the heart, cardiomyocyte apoptosis assays and gut microbiota analysis were detected. Left ventricular end-diastolic volume and diameter (LVd and LVDd), and left ventricular end-systolic volume and diameter in the HF+SR group were significantly enlarged compared with those in the HF group (all $P<0.05$ ). The HF+S group had a smaller LVDd and LVd than the HF+SR group (5.01 \pm 0.67 vs. $6.13 \pm 0.45 \mathrm{~mm}, P=$ $0.033 ; 121.44 \pm 38.74$ vs. $189.94 \pm 31.42 \mu \mathrm{L}, P=0.033)$. The messenger RNA and protein expression of galectin-3 and the number of apoptotic cardiomyocytes increased significantly in the HF+SR group compared to the HF group. Gut microbiota analysis showed that spermidine antagonists reduced the Firmicutes/Bacteroidetes ratio and changed the microbial community richness and diversity. In conclusion, spermidine can improve cardiac function in HF, and the regulation of gut microbiota and cardiac fibrosis may be a factor in the effect of spermidine on the improvement of cardiac function.

Keywords: heart failure, spermidine, microbiota, cardiac fibrosis, galectin-3

\section{INTRODUCTION}

Cardiac function and gut microbiota can affect each other. Reduced cardiac output causes intestinal wall ischemia, edema and structural disruption of the intestinal epithelial barrier function in heart failure (HF), leading to increased intestinal permeability, which in turn, contributes to the progression of HF (1). Furthermore, the impairment of intestinal barrier function leads to the translocation of gut bacterial deoxyribonucleic acid (DNA) and/or endotoxins into the bloodstream (2) and contributes to the deterioration of cardiac function (3). Studies have shown that in chronic HF, the gut microbiota is characterized by large compositional shifts with low bacterial richness and depletion of the core microbiota $(4,5)$. Therefore, the intestinal microbiota plays an important role in the development and progression of HF (6-8). 
Spermidine is a natural polyamine present in all living organisms at levels from micromolar to millimolar that is critically involved in the maintenance of cellular homeostasis (9, 10), usually with endogenous spermidine concentrations decreasing during the natural process of organismal aging $(11,12)$. Some studies have shown that dietary spermidine protects against cardiovascular aging (13), prevents cardiac hypertrophy and delayed the progression to HF (14), suggesting a beneficial effect on HF. One possible mechanism is that spermidine can enhance cardiac autophagy, mitophagy and mitochondrial respiration $(14,15)$. Moreover, the gut microbiota is significantly related to the synthesis of spermidine (16) and some gut microorganisms contain spermidine synthase (17). Spermidine can also enhance the gut barrier integrity and alter the gut microbiota in diet-induced obese mice (18). Although spermidine and the gut microbiota are both associated with $\mathrm{HF}$, it is not clear whether cardiac function can be affected by spermidine through modulating the gut microbiota when HF occurs. We speculated that regulation of the gut microbiota might be a contributing factor to the effect of spermidine on the improvement of cardiac function. A close relationship may exist among spermidine, HF and the gut microbiota, and the cardioprotective role of spermidine, especially to $\mathrm{HF}$, may be mediated by optimizing the gut microbiota composition.

In this study, we investigated the effect of spermidine on HF by altering its levels and assessed the association between spermidine and gut microbiota in an HF model induced by transverse aortic constriction (TAC) to explore whether spermidine can affect cardiac function by modulating the gut microbiota.

\section{MATERIALS AND METHODS}

\section{Aortic Constriction}

TAC was performed as described previously (19). Briefly, female Kunming mice, weighing 45-52 g, 11 weeks old, after anesthetization with pentobarbital sodium $(40 \mathrm{mg} / \mathrm{kg}$, intraperitoneally injected), were intubated and ventilated with a small animal respirator, at a rate of 130 breaths/min and a tidal volume of $0.4 \mathrm{ml}$. Aortic constriction was applied by tying a 7.0 silk string ligature around a 27 -gauge needle and then removing the needle. Sham-operated mice served as controls and were subjected to the same surgeries except for the ligation of the aorta. These experiments conformed to the Guide for the Care and Use of Laboratory Animals published by the US National Institutes of Health (NIH publication No. 85-23, revised 2011). The protocol was approved by the institutional ethics committee of Guangzhou First People's Hospital.

The HF mice were then randomly divided into three groups at 70 days post-operation: the HF group $(n=5)$, treated with saline; the $\mathrm{HF}+\mathrm{S}$ group $(n=8)$, administered with spermidine by intraperitoneal injection of $10 \mathrm{mg} / \mathrm{kg} / \mathrm{d}$ (Sigma-Aldrich, USA, diluted with saline solution) for 7 days (20); and the HF+SR group $(n=8)$, treated with trans-4-methylcycloh exylamine (4-MCHA), an antagonist of spermidine, by intraperitoneal injection of $100 \mathrm{mg} / \mathrm{kg} / \mathrm{d}$ (Sigma-Aldrich, USA, diluted with saline solution) for 7 days (21). All animals had free access to common food and water. At 70 days after administration, the mice were sacrificed by pentobarbital sodium overdose (150 $\mathrm{mg} / \mathrm{kg}$ ), and then fecal samples and left ventricular tissues were harvested for the analysis (Figure 1).

\section{Quantitative Real-Time Polymerase Chain Reaction}

Total ribonucleic acid (RNA) was isolated from left ventricular biopsies using TRIzol reagent (Invitrogen) according to the manufacturer's protocol. Primers were designed to detect galectin-3 gene expression (forward: GAGTACTAGAAGCGGCCGAG, reverse: CTGTGCCGCTCACCTGATTA) based on the sequences available in NCBI database (at http://ncbi.nlm.nih.gov) using Primer software. After measuring RNA concentration, $1.5 \mu \mathrm{g}$ RNA was treated with DNase I (Invitrogen) and used for cDNA synthesis by reverse transcriptase M-MLV (Takara, Japan). The galectin-3 messenger RNA (mRNA) levels were measured with CFX96 quantitative real-time polymerase chain reaction (QT-PCR) system (Bio-Rad). The relative amounts of mRNA were determined based on $2-\Delta \Delta \mathrm{Ct}$ calculations.

\section{Echocardiography}

Transthoracic echocardiography was performed using a Vevo 2100 imaging system equipped with a $15-30 \mathrm{MHz}$ linear array transducer (VisualSonics, Inc., Canada) by a single blinded observer as described previously (14). The following parameters were measured and averaged from 3 cardiac cycles: stroke volume, left ventricular end-diastolic volume and diameter (LVVd and LVDd), and left ventricular end-systolic volume and diameter (LVVs and LVDs), left ventricular anterior and posterior wall thickness in systole and diastole (LVAWs, LVAWd, LVPWs, and LVPWd). The left ventricular mass was calculated following the previously described method of Gao et al.: left ventricular mass $=\left[(\text { LVDd }+ \text { LVAWd }+ \text { LVPWd })^{3}-\right.$ LVDd 3] $\times 1.055$, where 1.055 is the gravity of the myocardium (22). Fractional shortening (FS, \%) was calculated using the equation: $100 \times[(\mathrm{LVDd}-\mathrm{LVDs}) / \mathrm{LVDd}]$. Left ventricular ejection fraction (LVEF, \%) was calculated as $100 \times(\mathrm{LVVd}-\mathrm{LVVs}) / \mathrm{LVVd}$.

\section{Western Blot Analysis}

Left ventricular tissues were homogenized in RIPA buffer (Beyotime Biotechnology, China) to obtain whole cell lysates and centrifuged to isolate the protein. Fifty micrograms of protein was subjected to SDS-polyacrylamide gel electrophoresis and blotted onto a PVDF membrane. After blocking with 5\% non-fat milk, the membranes were incubated with the following primary antibodies: galectin-3 (Abcam, Shanghai, China), spermidine (Abcam, Shanghai, China) or $\beta$-actin (Proteintech, Wuhan, China). Then, after incubation with anti-rabbit HRP-conjugated IgG (1:2,500, Boster, China) or anti-mouse HRP-conjugated IgG (1:2,500, Boster, China) for $1 \mathrm{~h}$ at room temperature, the immunoreactive bands were visualized by chemiluminescence reagents (ECL; KeyGEN BioTECH, Nanjing, China). The band intensity was quantified using ImageJ analysis software in a blinded manner, and all bands were normalized to the corresponding $\beta$-actin bands. 


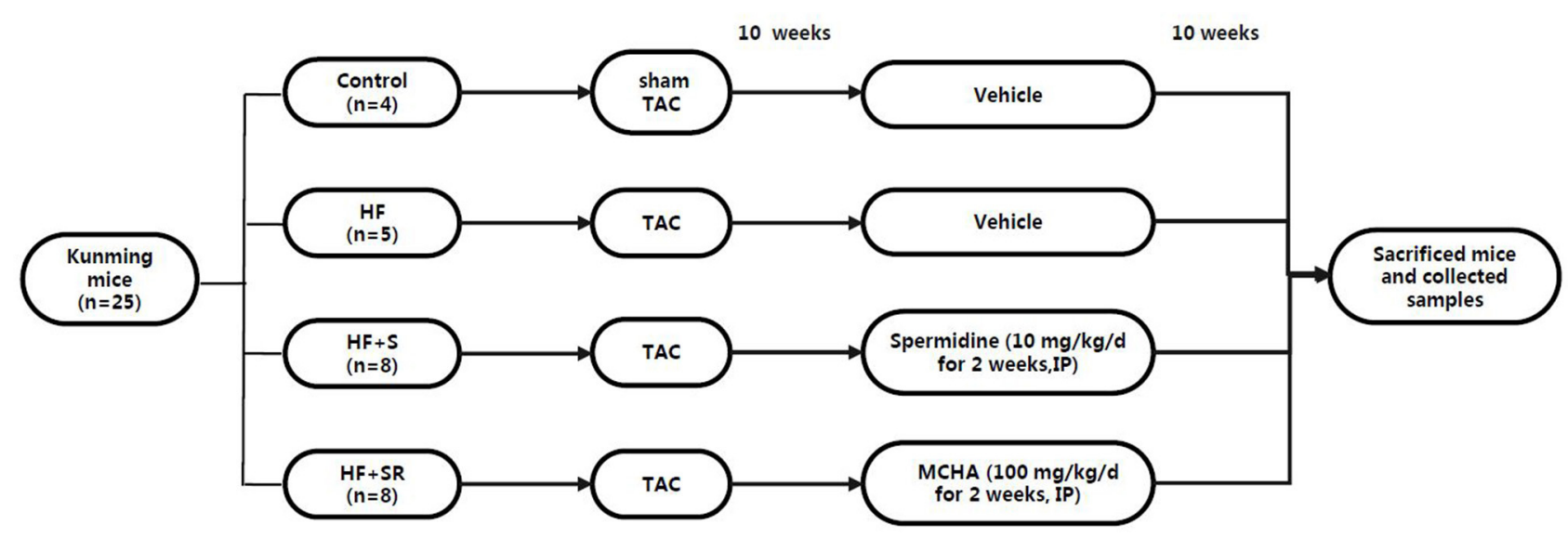

FIGURE 1 | The animal grouping and time line of the experimental protocol.

\section{Immunohistochemistry}

For galectin-3 and spermidine immunohistochemistry, formalinfixed left ventricular tissues were embedded in paraffin and sectioned into 5- $\mu \mathrm{m}$-thick sections. After deparaffinization, rehydration and pre-treatment with hydrogen peroxide, the sections were incubated with primary antibodies against galectin-3 (Abcam, Shanghai, China) and spermidine (Abcam, Shanghai, China) overnight at $4^{\circ} \mathrm{C}$ before being incubated with a secondary antibody for $1 \mathrm{~h}$ at room temperature. The sections were stained with DAB (Servicebio, China) followed by counterstaining with hematoxylin. Three entire sections per heart were examined by skilled observers blinded to the treatment group.

\section{Terminal Deoxynucleotidyl Transferase-Mediated dUTP Nick-End Labeling Staining}

TUNEL assays were performed to detect apoptotic cells in the heart tissue sections according to the manufacturer's protocols (Roche, China). Briefly, the left ventricular tissues were deparaffinized and rehydrated with serial changes in xylene and five different concentrations of ethanol. After proteinase $\mathrm{K}$ and endogenous peroxidase treatment, the heart tissue sections were stained with DAB (Servicebio, China). The TUNEL-positive cells were quantified using Image-Pro Plus analysis software.

\section{Microbiome Sequencing and Analysis}

Mouse fecal samples were collected under sterile conditions by pressing on the outer wall of the colon to push its contents into sterile tubes, at which time they were snap frozen in liquid nitrogen and then stored at $-80^{\circ} \mathrm{C}$ until analysis. Total genomic DNA from the samples was extracted using the CTAB/SDS method by Novogene (Tianjin, China). The DNA concentrations and purity were analyzed on $1 \%$ agarose gels. The diluted DNA was used as the template to amplify the 16S rRNA (16S V4: 515F-806R) with specific barcoded primers. All polymerase chain reaction (PCR) reactions were carried out with Phusion ${ }^{\circledR}$ HighFidelity PCR Master Mix (New England Biolabs). The PCR products were purified with the GeneJET Gel Extraction Kit (Thermo Scientific). The sequencing libraries were generated using the NEB Next ${ }^{\circledR}$ UltraTM DNA Library Prep Kit for Illumina (NEB, USA) and index codes were added. The library quality was assessed on the Qubit@ 2.0 Fluorometer (Thermo Scientific) and the Agilent Bioanalyzer 2100 system. Then the qualified library was sequenced on the Illumina MiSeq platform, and $250 \mathrm{bp}$ paired-end reads were generated. The paired-end reads were combined using FLASH (V1.2.7) and assigned to the samples according to their barcodes.

Sequence analysis was conducted by Uparse software (V7.0.1001) and sequences with $\geq 97 \%$ similarity were assigned to the same operational taxonomic units (OTUs). Alpha diversity was analyzed through 3 parameters, including Chao 1 , abundance coverage estimator (ACE), observed- species and phylogenetic diversity (PD)-whole tree. The linear discriminant analysis (LDA) effect size (LEfSe) was selected for the quantitative analysis of the microbial biomarkers within the different groups. The differences between the groups were assessed according to the LDA scores [OTUs with $(\log 10)]$. Cluster assessment was preceded by principal component analysis (PCA) using QIIME software (V1.7.0).

\section{Statistical Analyses}

Continuous variables were expressed as the mean \pm standard deviation. Differences among the means were evaluated with a two-independent-sample $t$-test or one-way ANOVA test with $\mathrm{S}-\mathrm{N}-\mathrm{K}$ analysis, as appropriate. $P$-values were two-sided and considered significant when $<0.05$. Statistical analyses were carried out using the SPSS version 17.0 software package (SPSS Inc., Chicago, USA).

\section{RESULTS}

\section{Assessment of the HF Model Induced by Aortic Constriction and Grouping}

During the observation period after the operation, three mice died, while all sham-operated animals (controls, $n=4$ ) survived. 
The TAC mice showed some signs of congestive HF such as anorexia, dyspnea and lethargy at 70 days post-operation, indicating the appearance of HF. Echocardiography showed that LVDs, LVDd, LVVs, LVVd, left ventricular mass average weight (LV Mass AW), and LV Mass AW (corrected) were significantly increased, and LVEF and FS were significantly decreased in the HF mice compared to the sham-operated mice (all $P<0.05$, Table 1).

\section{Variation in Echocardiographic Parameters After the Administration of Spermidine or Its Antagonist}

After administration of spermidine or 4-MCHA, the HF mice were examined by Doppler echocardiography. Figure 2 shows that the echocardiographic parameters, including LVDs, LVDd, LVVs, LVVd, FS, LVEF, LV mass AW, and LV mass AW (corrected) were not significantly different at 14 days after administration among the three groups: the $\mathrm{HF}, \mathrm{HF}+\mathrm{S}$, and $\mathrm{HF}+\mathrm{SR}$ groups. However, statistically significant differences were noted 56 days after administration. The LVDs, LVDd, LVVs, and LVVd in the HF+SR group were greatly enlarged (5.51 \pm 0.69 vs. $4.14 \pm 0.47 \mathrm{~mm}, P=0.033 ; 6.13 \pm 0.45$ vs. $5.02 \pm$ $0.40 \mathrm{~mm}, P=0.019 ; 150.44 \pm 41.68$ vs. $76.92 \pm 21.08 \mu \mathrm{L}, P$ $=0.040 ; 189.94 \pm 31.42$ vs. $119.83 \pm 22.31 \mu \mathrm{L}, P=0.022)$ compared with those in the HF group. Furthermore, LVDd and LVVd were significantly smaller $(5.01 \pm 0.67$ vs. $6.13 \pm$ $0.45 \mathrm{~mm}, P=0.033 ; 121.44 \pm 38.74$ vs. $189.94 \pm 31.42 \mu \mathrm{L}$, $P=0.033)$ in the $\mathrm{HF}+\mathrm{S}$ group than in the $\mathrm{HF}+\mathrm{SR}$ group (Figure 3).

\section{The mRNA Expression of Galectin-3 in the Heart}

Relative gene expression of galectin-3 in the left ventricular tissues was analyzed using QT-PCR at 70 days after administration. The results showed that galectin-3 expression was significantly increased in the two treated groups. The galectin-3 mRNA levels increased 3.5-fold in the HF+S group and 3.1-fold in the HF+SR group compared to the HF group (both $P<0.05$ ). However, there was no difference between the $\mathrm{HF}+\mathrm{S}$ and $\mathrm{HF}+\mathrm{SR}$ groups (Figure 4A).

\section{The Protein Expression of Galectin-3 in the Heart}

To investigate the protein expression of galectin-3 in the heart, western blotting was conducted. The results showed that the expression levels of spermidine were elevated in the $\mathrm{HF}+\mathrm{S}$ group, and the relative protein expression levels of galectin-3 were higher in the $\mathrm{HF}+\mathrm{SR}$ group than in the $\mathrm{HF}$ and $\mathrm{HF}+\mathrm{S}$ groups ( $\sim 2.1$-fold, all $P<0.05)$. However, no difference was observed in the protein expression of galectin-3 between the $\mathrm{HF}$ and $\mathrm{HF}+\mathrm{S}$ groups (Figures 4B,C).

\section{Immunohistochemistry of Galectin-3 in the Heart Tissue}

The protein expression of galectin-3 in the heart was also confirmed by immunohistochemistry and semiquantitative analysis, indicated as the values of the average optical density (AOD) measured by Image-Pro Plus 6.0. Immunohistochemistry staining showed that the protein expression of galectin-3 was significantly increased in the $\mathrm{HF}+\mathrm{SR}$ group compared with the control and HF groups (AOD, $0.051 \pm 0.005$ vs. $0.040 \pm$ 0.005 and $0.043 \pm 0.005$, both $P<0.05$ ). There was also no difference in the expression of galectin-3 between the HF and $\mathrm{HF}+\mathrm{S}$ groups (AOD, $0.043 \pm 0.005$ vs. $0.043 \pm 0.010, P=0.976$ ) (Figure 5).

\section{Effect of Spermidine on Cardiomyocyte Apoptosis}

To assess the effect of spermidine on cardiomyocyte apoptosis, a TUNEL assay was used. Brown-stained cells accompanied by condensed or fragmented nuclei were considered TUNELpositive cells. The number of apoptotic cardiomyocytes was significantly increased in the $\mathrm{HF}, \mathrm{HF}+\mathrm{S}$ and $\mathrm{HF}+\mathrm{SR}$ groups compared with the controls. There was a modest 0.5 -fold increase in the number of apoptotic cells in the $\mathrm{HF}+\mathrm{SR}$ group compared to the HF group, but no difference was observed between the $\mathrm{HF}$ and $\mathrm{HF}+\mathrm{S}$ groups (Figure 6).

\section{Compositional Alteration of Gut Microbiota in Mice With Different Treatment Schemes}

The compositions of the microbial community richness or diversity were assessed by ACE, observed-species and PD-whole

TABLE 1 | Comparisons of echocardiographic parameters between sham-operated and heart failure groups at 10 weeks post-operation and before administration.

\begin{tabular}{|c|c|c|c|c|c|c|c|c|c|}
\hline & $\begin{array}{c}\text { Heart rate } \\
\text { (bpm) }\end{array}$ & LVDs (mm) & LVDd (mm) & LVVs (uL) & LVVd (uL) & FS (\%) & LVEF (\%) & $\begin{array}{c}\text { LV mass AW } \\
(\mathrm{mg})\end{array}$ & $\begin{array}{c}\text { LV mass AW } \\
\text { corrected } \\
(\mathrm{mg})\end{array}$ \\
\hline Sham $(n=4)$ & $438 \pm 35$ & $2.54 \pm 0.56$ & $4.12 \pm 0.54$ & $\begin{array}{c}24.69 \pm \\
13.71\end{array}$ & $\begin{array}{c}76.63 \pm \\
24.48\end{array}$ & $38.79 \pm 5.32$ & $69.29 \pm 7.12$ & $\begin{array}{c}138.03 \pm \\
29.41\end{array}$ & $\begin{array}{c}110.42 \pm \\
23.53\end{array}$ \\
\hline $\mathrm{HF}(n=21)$ & $499 \pm 40$ & $3.53 \pm 0.47$ & $4.89 \pm 0.42$ & $\begin{array}{c}53.45 \pm \\
17.04\end{array}$ & $\begin{array}{c}113.38 \pm \\
23.67\end{array}$ & $27.89 \pm 5.98$ & $53.39 \pm 9.12$ & $\begin{array}{c}218.56 \pm \\
46.25\end{array}$ & $\begin{array}{c}174.84 \pm \\
37.00\end{array}$ \\
\hline$P$ & 0.008 & 0.004 & 0.011 & 0.012 & 0.023 & 0.008 & 0.010 & 0.010 & 0.010 \\
\hline
\end{tabular}

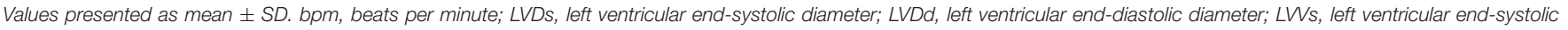
volume; LWd, left ventricular end-diastolic volume; FS, fractional shortening; LVEF, left ventricular ejection fraction; LV Mass AW, left ventricular mass average weight. 
A

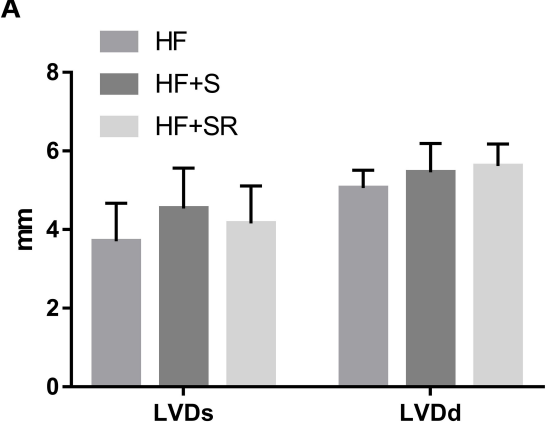

C

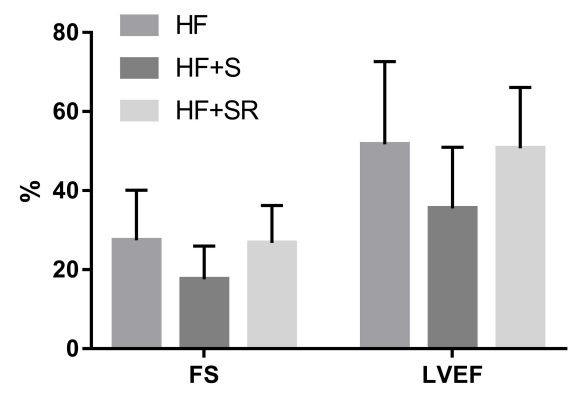

B

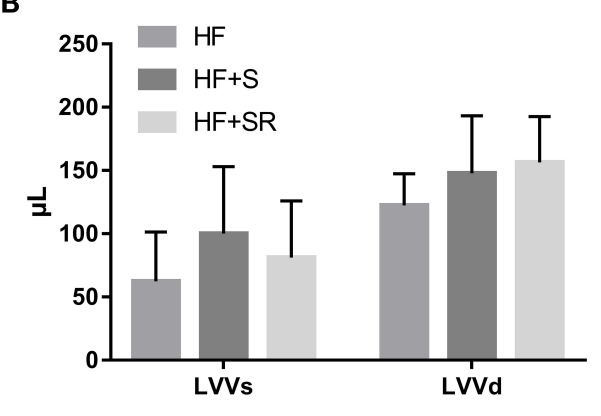

D

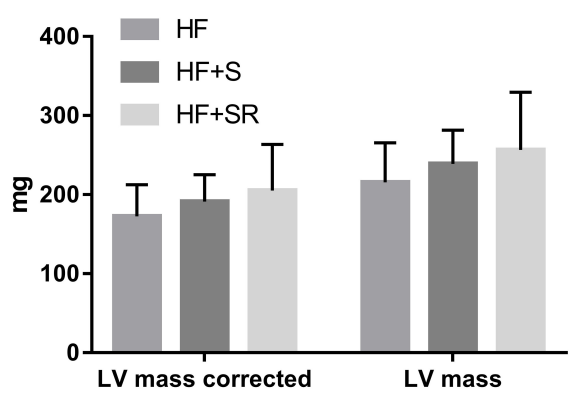

E

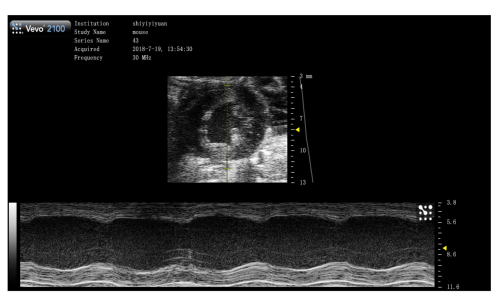

F

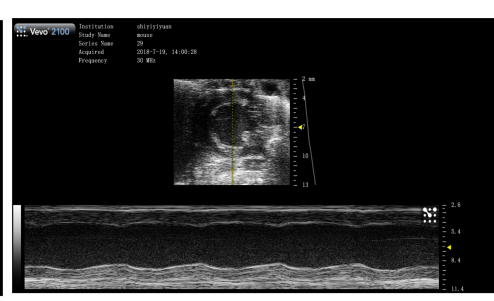

G

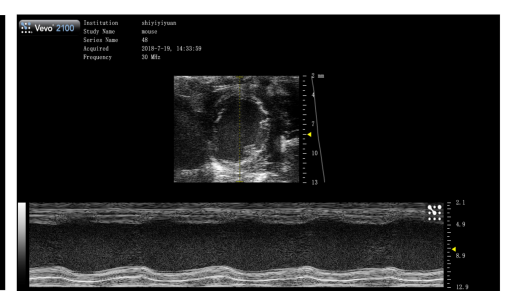

FIGURE 2 | Difference in the echocardiographic parameters among the HF $(n=5), \mathrm{HF}+\mathrm{S}(n=8)$ and HF+SR $(n=8)$ mice at 14 days after administration. There were no differences in LVDs, LVDd (A), LWs, LVd (B), FS, LVEF (C), LV mass or LV mass corrected (D) among the three groups. Representative echocardiographic images showing cardiac function and dimensions in the HF (E), HF+S (F), and HF+SR (G) mice.

tree. The ACE and observed-species index were significantly higher in the controls than in the $\mathrm{HF}, \mathrm{HF}+\mathrm{S}$ or $\mathrm{HF}+\mathrm{SR}$ groups (Figures 7A,B, all $P<0.05$ ). The $\mathrm{PD}$-whole tree index was lower only in the $\mathrm{HF}+\mathrm{S}$ group than in the controls (Figure 7C, $P=$ $0.023)$. Moreover, a significant difference in unweighted UniFrac distances was observed between these two groups $(P=0.038$; Figure 7D).

LefSe analysis showed that at the family and genus levels, the $\mathrm{HF}+\mathrm{S}$ mice had an increased abundance of Muribaculaceae, whereas increased abundances of Tannerellaceae, Beijerinckiaceae, Bacteroidaceae, Parabacteroides and Bacteroides were found in the HF mice (Figure 7E). When compared to the HF mice, the $\mathrm{HF}+\mathrm{SR}$ mice possessed more Muribaculaceae, but no other difference was found between the two groups.
MetaStat analysis showed that the spermidine antagonist reduced the relative abundance of Millionella massiliensis and other unidentified bacteria (both $P<0.05$, Figure 8A).

By comparison with the top 35 most frequent bacterial genera, the HF mice had 5 abundant genera (red color) within Bacteroidetes and 4 rare genera (blue color) within Firmicutes, and the $\mathrm{HF}+\mathrm{S}$ mice possessed 3 abundant genera within Firmicutes and 4 rare genera within Bacteroidetes, whereas the $\mathrm{HF}+\mathrm{SR}$ mice had 2 abundant genera within Bacteroidetes and 4 rare genera within Firmicutes (Figure 8B).

As shown in Figure 8C, all tested mice dominated two major microbiota among the top 10 phyla: Bacteroidetes (54-74\%) and Firmicutes (19-40\%). However, the Firmicutes/Bacteroidetes ratio was different. The HF+SR mice had more Bacteroidetes and less Firmicutes. 


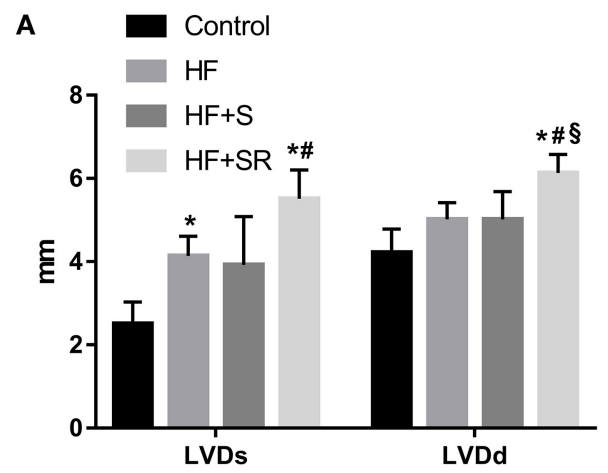

C

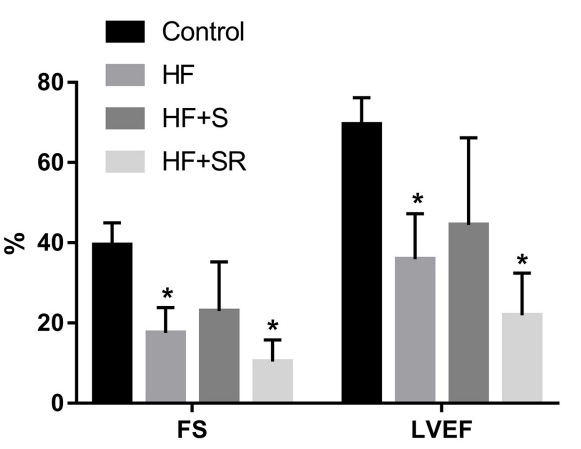

E

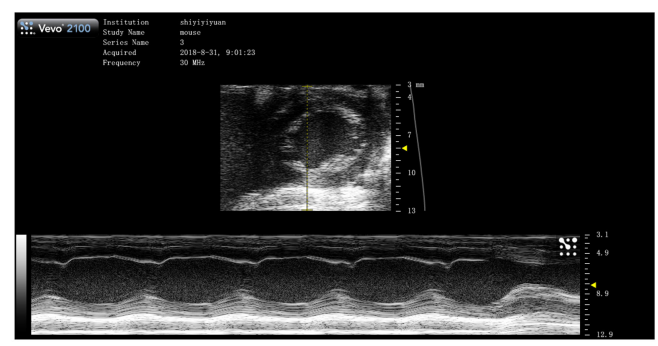

G

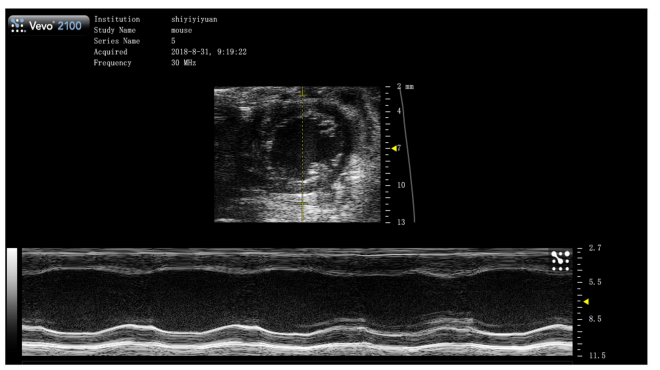

B

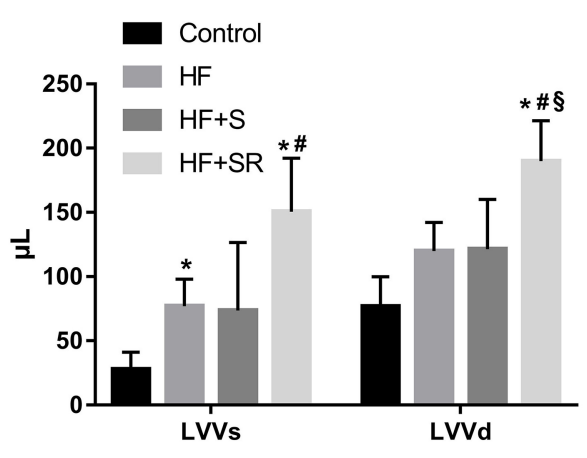

D

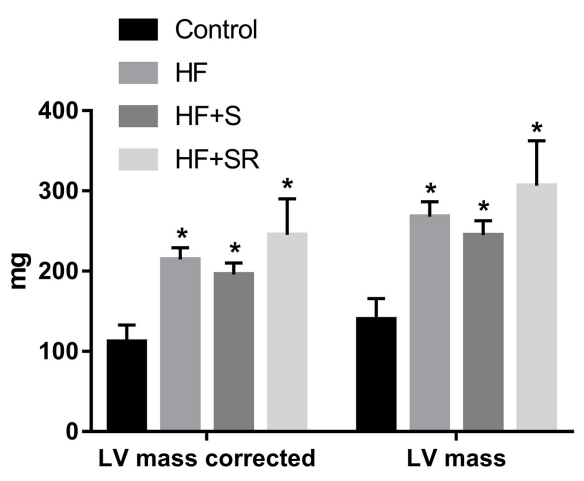

$\mathbf{F}$

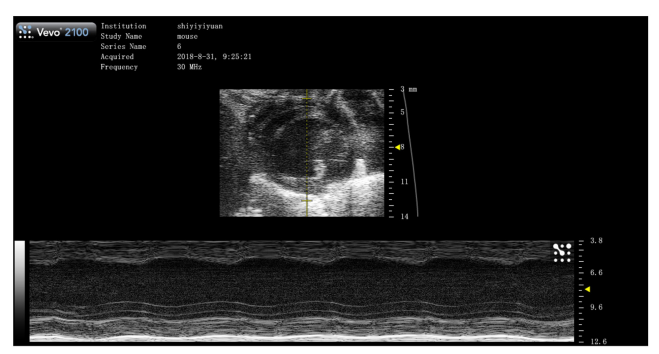

H

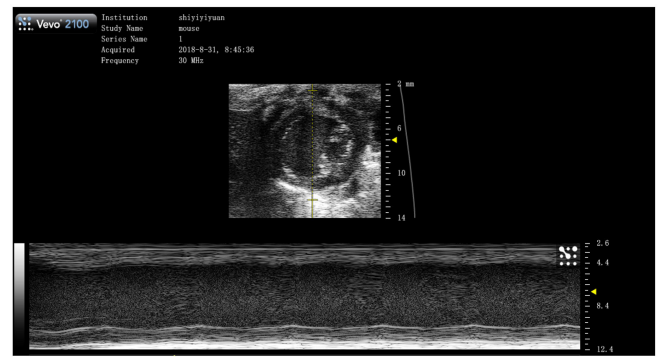

FIGURE 3 | Comparison of echocardiographic parameters among the different groups at 56 days after administration. Comparisons of LVDs, LVDd (A), LVs, LVd (B), FS, LVEF (C), LV mass and LV mass corrected (D) among the control $(n=4), \mathrm{HF}(n=5), \mathrm{HF}+\mathrm{S}(n=8)$, and HF+SR $(n=8)$ mice. Representative echocardiographic images in the control (E), HF (F), HF+S (G), and HF+SR (H) mice. ${ }^{*} P<0.05$ vs. controls, $\# P<0.05$ vs. HF group, and ${ }^{\S} P<0.05$ vs. HF+S group.

\section{DISCUSSION}

In the present study, the $\mathrm{HF}+\mathrm{SR}$ mice presented worsening echocardiographic parameters including LVDs, LVDd, LVVs, and LVVd compared with the HF mice. LVDd and LVVd were better in the $\mathrm{HF}+\mathrm{S}$ mice than in the $\mathrm{HF}+\mathrm{SR}$ mice. The mRNA and protein expression levels of galectin-3 in the heart were significantly higher in the $\mathrm{HF}+\mathrm{SR}$ mice than in the 

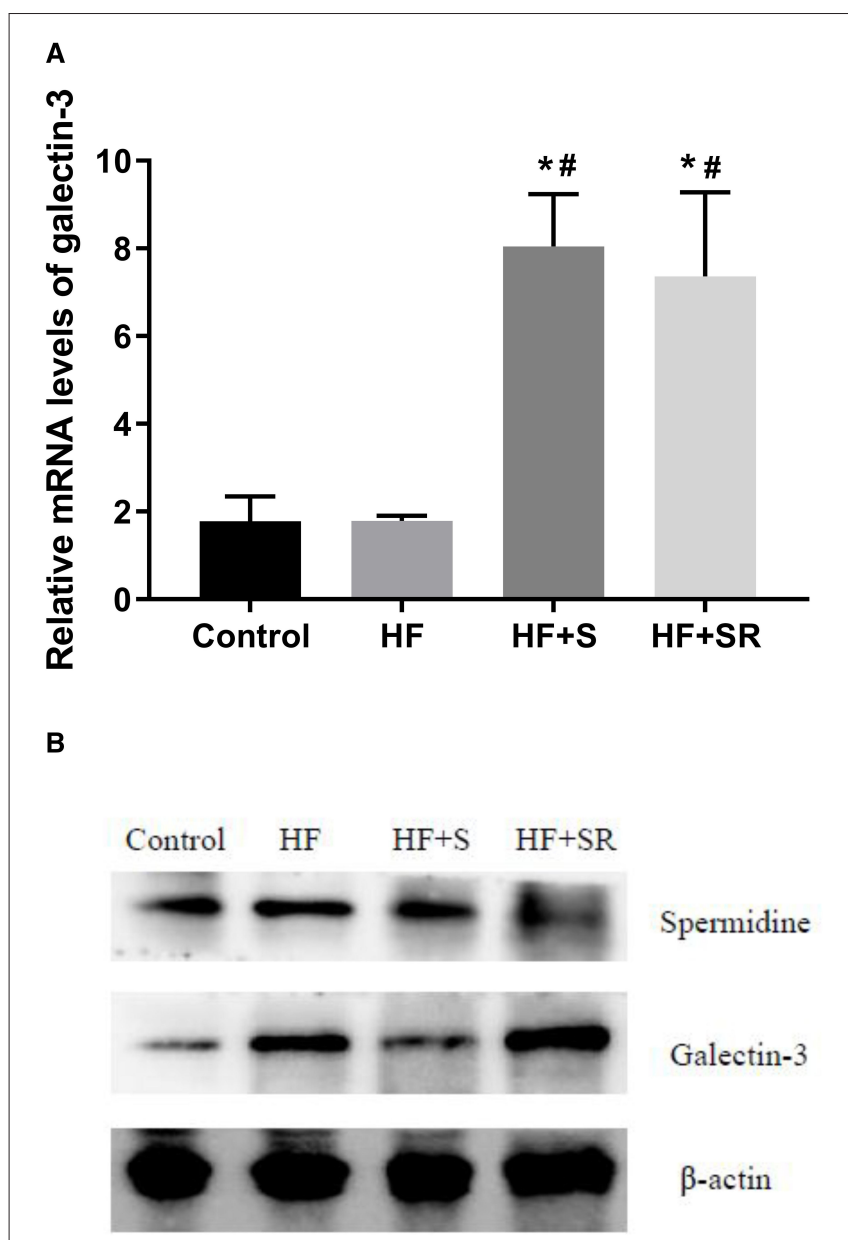

C

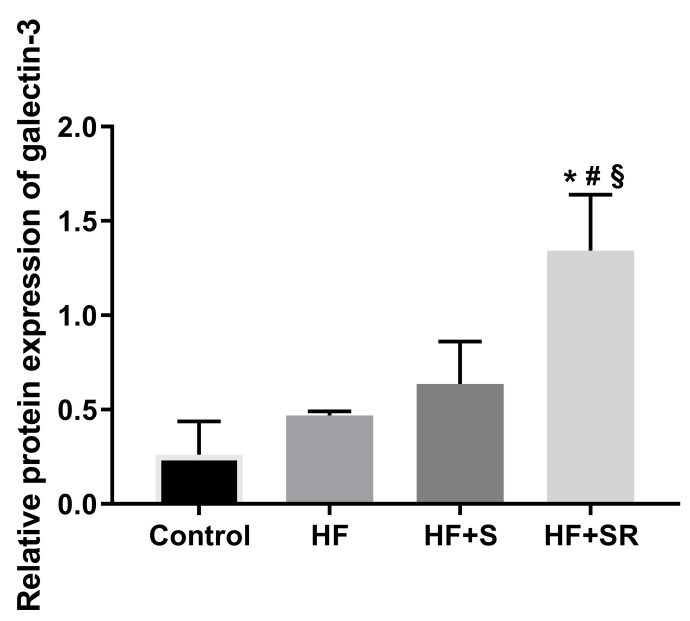

FIGURE 4 | The mRNA and protein expression of galectin-3 in the heart in different groups. Relative mRNA levels of galectin-3 in the heart (A). Representative immunoblots for galectin-3 in the heart (B). Quantitation of galectin-3 protein levels in the heart (C). ${ }^{*} P<0.05$ vs. controls $(n=4)$, \#P< 0.05 vs. HF group $(n=5)$ and ${ }^{\S} P<0.05$ vs. HF+S group $(n=8)$.
HF mice. In addition, the amount of cardiomyocyte apoptosis was the highest in the mice treated with an antagonist of spermidine. Analysis of the gut microbiota showed that the alpha diversity was significantly higher in the controls than in the HF, HF $+\mathrm{S}$, or $\mathrm{HF}+\mathrm{SR}$ groups. Moreover, the microbial community diversity decreased significantly and the microbial composition changed considerably after administration of the spermidine antagonist, especially decrease of Millionella massiliensis, the Firmicutes/Bacteroidetes ratio and the increase of Muribaculaceae. These findings showed that inhibiting spermidine deteriorated cardiac function and that increasing spermidine was beneficial to cardiac function in HF, and the regulation of the gut microbiota might be a contributing factor to the effect of spermidine on cardiac function.

Dietary spermidine protects against cardiovascular aging (23). In rats with hypertension-induced congestive heart failure, spermidine feeding decreased their systolic blood pressure and prevented cardiac hypertrophy, thus delaying the progression to HF. In humans, high levels of dietary spermidine increase survival and are inversely related to the incidence of cardiovascular disease $(13,14)$. These results implied that supplementation with spermidine might prevent the occurrence of cardiovascular diseases including HF. However, how was HF affected when changing the levels of spermidine? Our results showed that in HF, reducing spermidine levels by administrating its antagonist led to worsening of the echocardiographic parameters and an increase of galectin-3, a marker of cardiac fibrosis (24), suggesting that decreasing spermidine levels can aggravate cardiac fibrosis and deteriorate cardiac function. Thus, spermidine may be helpful in alleviating cardiac fibrosis during the process of HF and may be beneficial for the recovery of cardiac function. Similar to other potential novel biomarkers for myocardial fibrosis such as secreted frizzled-related protein $(25,26)$, spermidine may have potential preventive or therapeutic value in myocardial fibrosis and HF.

Spermidine plays many important roles in many aspects of cardiovascular pathophysiology. It can reverse arterial stiffness and restore arterial endothelial function in old mice (27). It reduces lipid accumulation and the formation of atherosclerotic plaques $(28,29)$. These results show that spermidine has a protective role to arteries and is beneficial for supplying cardiocytes with oxygen and nutrients and maintaining cardiac function. Therefore, maintenance of the appropriate spermidine levels may be important for maintaining cardiac function.

Our results showed that a decrease in spermidine reduced cardiac function, and supplementation with spermidine had a beneficial effect on cardiac function. Although the galectin-3 mRNA levels increased after spermidine supplementation, an increase in protein levels was not observed. Post-transcriptional regulation and translation of mRNA proteins may be responsible for the discrepancy in mRNA and protein levels in galectin3. The method and duration of spermidine supplementation may be one cause of the small beneficial effect of spermidine supplementation in this study, because the absorption rate may be different when spermidine is administered by injection 


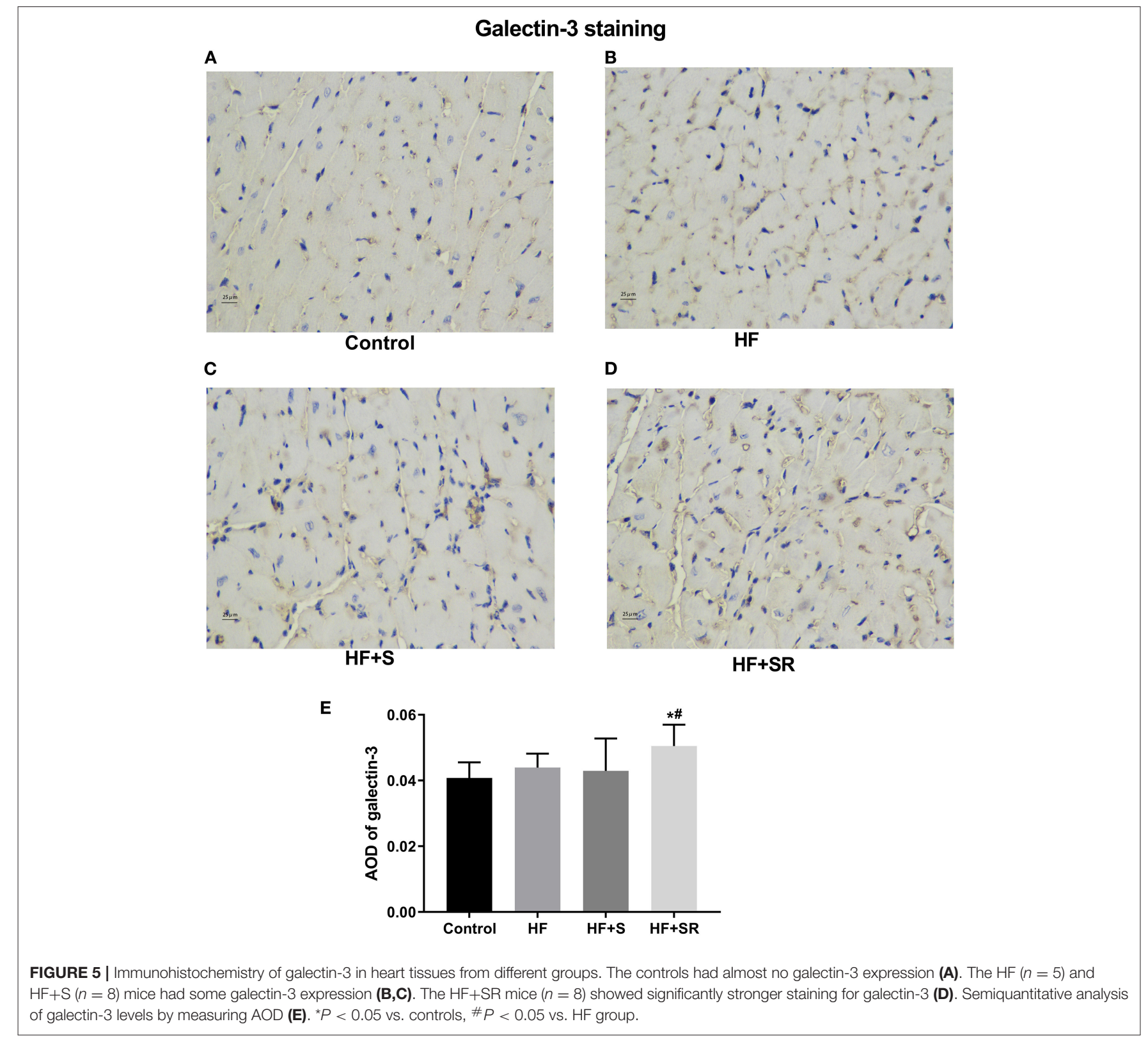

or orally. In addition, spermidine is a two-edged sword, and only appropriate concentrations can improve cardiac function because inappropriate spermidine levels damage tissue and organs. In rats, spermidine had a potential antipsychotic effect at a low dose $(10 \mathrm{mg} / \mathrm{kg})$, but adverse effects appeared at a higher dose $(20 \mathrm{mg} / \mathrm{kg})$ (30). It has been reported that excessive intake of spermidine is not without risk. Spermidine showed dose-dependent cytotoxicity in the cultured cells via necrosis and was found to be toxic when its concentrations were above the maximum at which they have been found in food (31). Cancer patients with higher levels of spermidine have a worsed prognosis suggesting that abundant spermidine likely contributes to enhanced growth rates of cancer cells because it is indispensable for cell growth and tumor progression $(7,32)$.
Moreover, spermidine is not associated with a protective role in cardiovascular disease. It also has an adverse effect on the cardiovascular system. Patients with higher metabolic scores calculated according to the metabolic profile including spermidine levels had worse New York Heart Association functional classes and an adverse prognosis (33). In addition, because cardiac dysfunction is often accompanied by microcirculation disorders (34), a sufficient supply of the myocardium with oxygen and energy by the microcirculation plays very important roles in maintaining normal cardiac function $(35,36)$. However, Wierich et al. thought that spermidine had no effect on the quantitative characteristics of capillaries or arterioles, including the capillary volume, surface area and length as well as vascular endothelial growth factor-A 

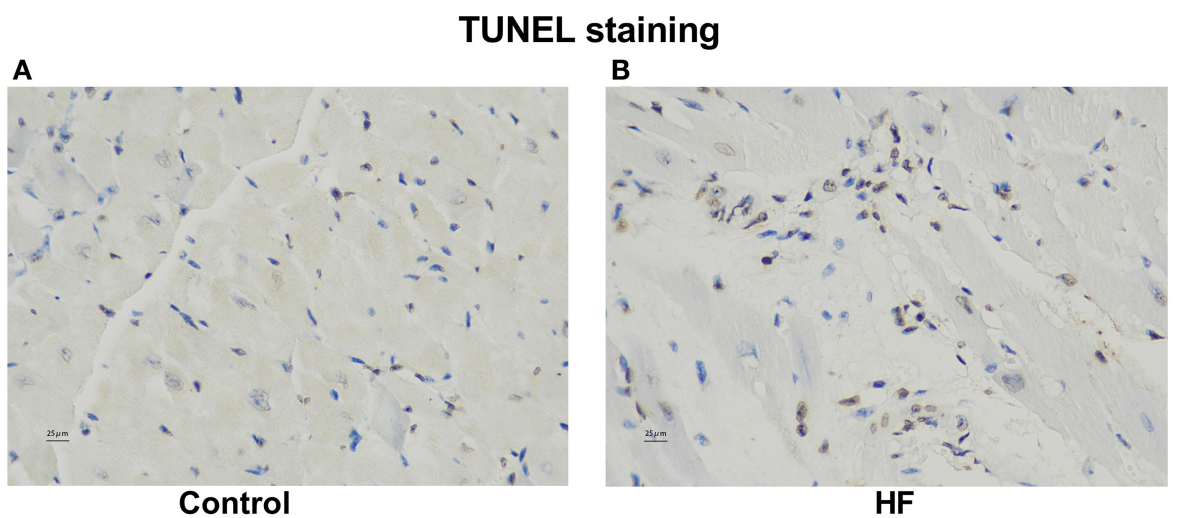

C

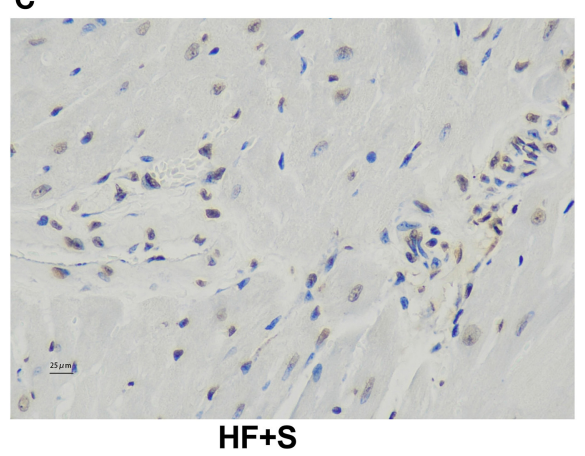

D

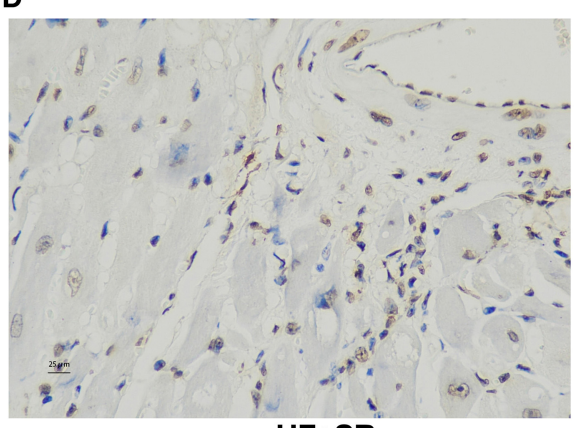

HF+SR

E

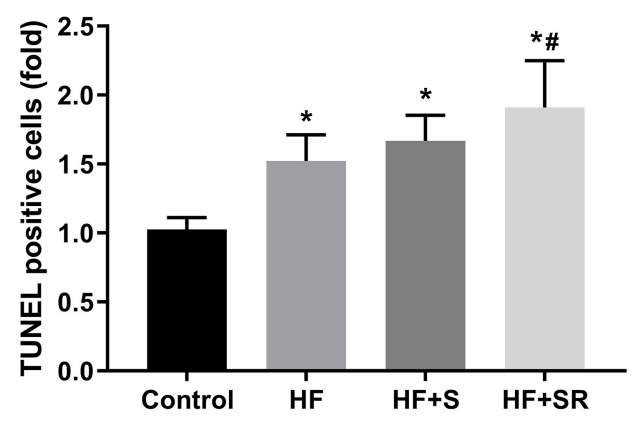

FIGURE 6 | Differences in the number of apoptotic cardiomyocytes among the four groups. Representative photomicrographs of ventricular tissue stained for TUNEL. The controls had very few TUNEL-positive cells (A). Many TUNEL-positive cells appeared in the HF $(n=5)$ and HF+S $(n=8)$ mice (B,C). The percentage of TUNEL-positive cells was significantly increased in the HF+SR mice $(n=8)(\mathbf{D})$. Quantitative analysis of TUNEL-positive cells in the heart sections $(\mathbf{E}){ }^{*} P<0.05$ vs. controls, $\# P<0.05$ vs. the HF group.

expression, suggesting that spermidine has no effect on the quantitative structural characteristics of the microcirculation in the aging heart (37). Therefore, the therapeutic role of spermidine may be dose-dependent and identifying a suitable concentration for the improvement of heart failure is very important.

For gut microbiota analysis, consistent with previous observations $(3,4)$, our results showed that the bacterial richness decreased when HF occurred, regardless of whether spermidine or its antagonist was administered. Furthermore, spermidine increased the abundance of Muribaculaceae and its antagonist significantly decreased the Firmicutes/Bacteroidetes ratio, a widely used marker of gut dysbiosis, meaning that reduced Firmicutes/Bacteroidetes ratio was related to deteriorated cardiac function. Firmicutes and Bacteroidetes are the two dominant phyla in the murine and human intestinal microbiota (38), and the Firmicutes/Bacteroidetes ratio was associated with many disorders. Elderly individuals have a lower Firmicutes/Bacteroidetes ratio than adults (39), and the Firmicutes/Bacteroidetes ratio decreased in isoproterenolinduced acute myocardial ischemia rats and chronic intermittent hypoxia exposed pigs $(40,41)$.

However, different results were also observed in other studies. Lataro et al. thought that the Firmicutes/Bacteroidetes ratio was not altered in HF model rats subjected to myocardial 

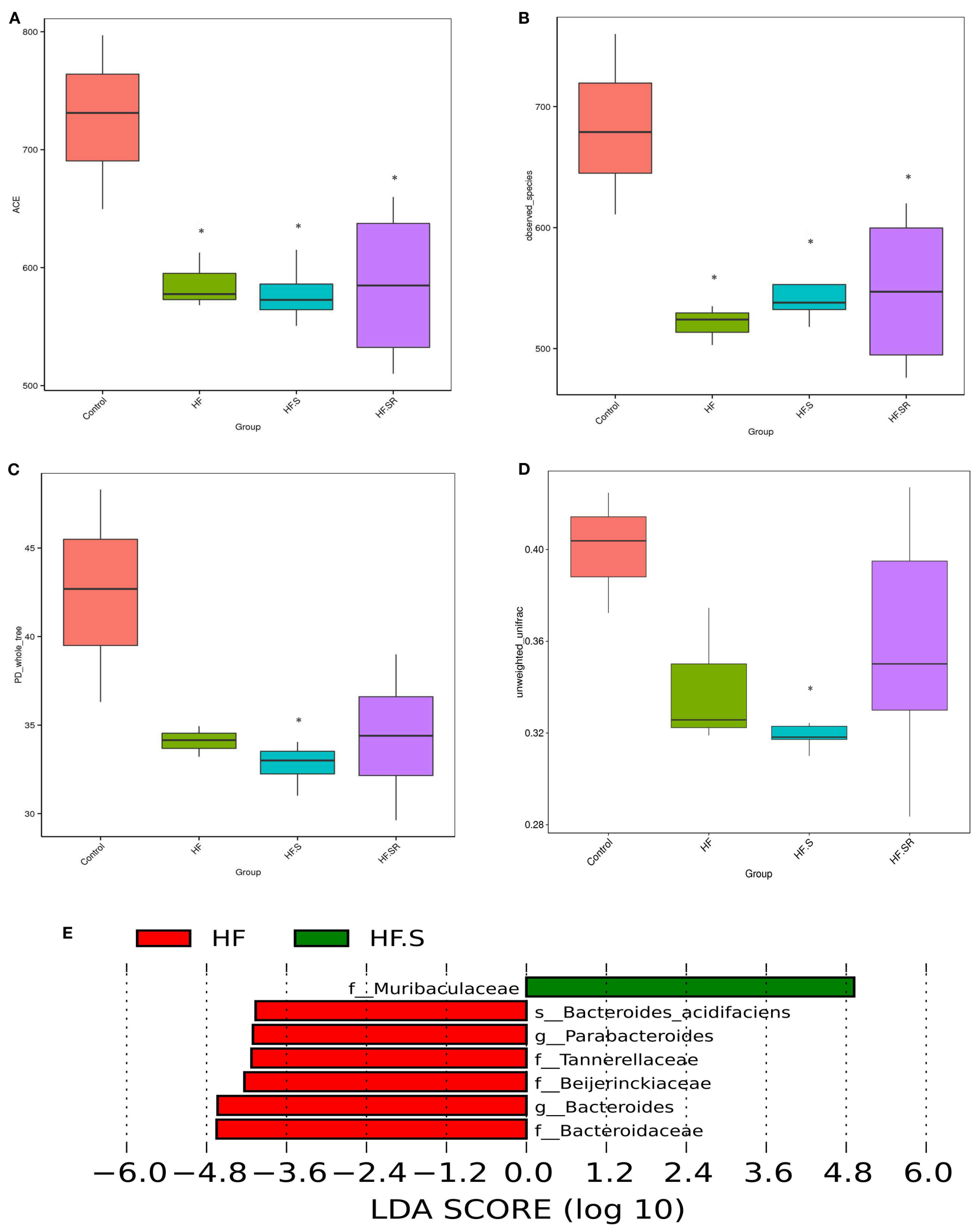

FIGURE 7 | The compositions of the microbial community richness or diversity in the different groups. The community richness was estimated by ACE (A) and the observed-species (B). The community diversity is represented by the PD-whole tree (C). Unweighted UniFrac distances revealed significant differences between the controls $(n=4)$ and HF+S mice $(n=8)$ (D). The relative abundance of 6 taxa decreased, while only one increased in the HF $+S$ mice compared with the HF mice $(n=$ 5) (E). ${ }^{*} P<0.05$ vs. controls. 


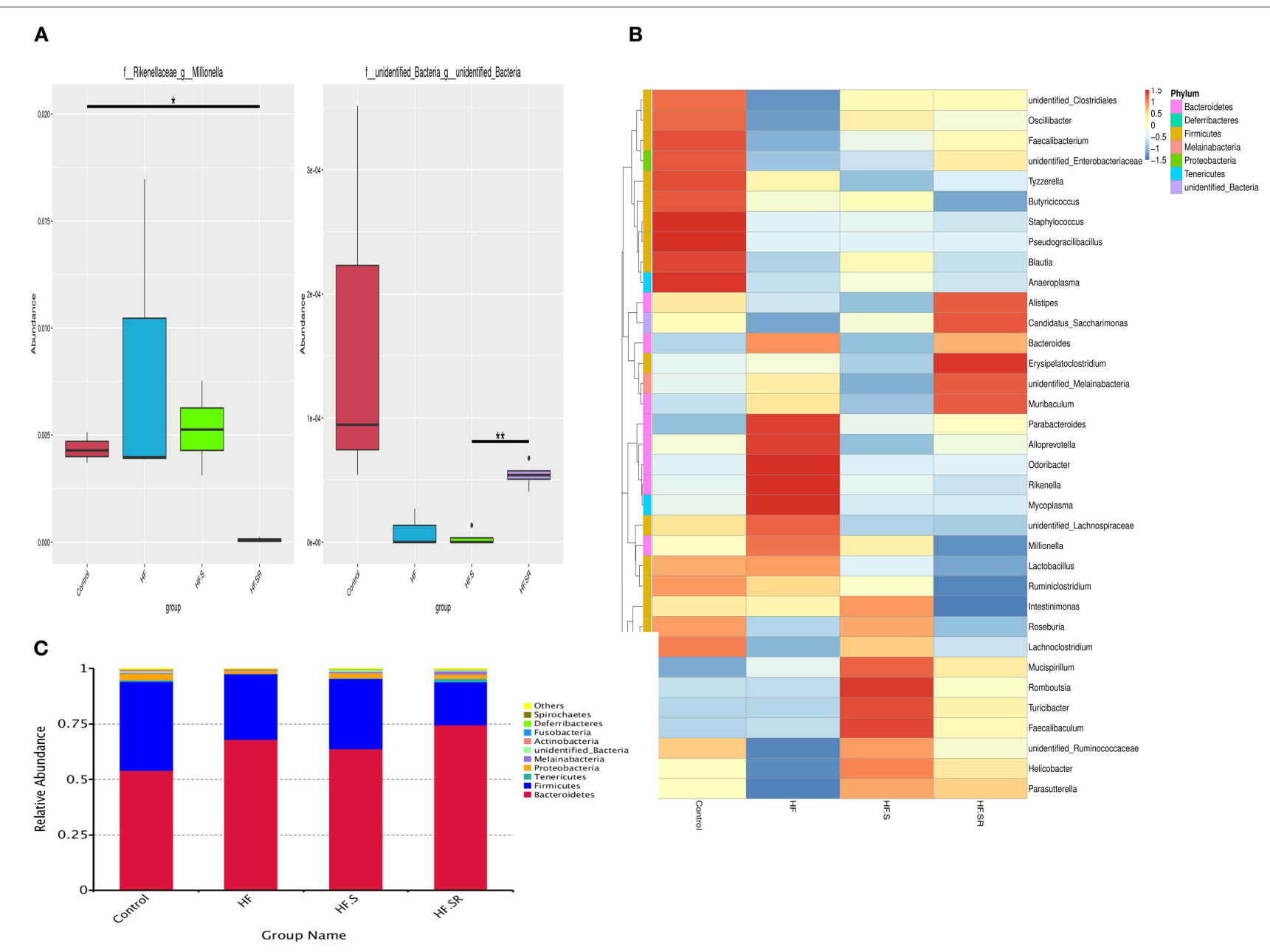

FIGURE 8 | Distribution of bacterial abundance. MetaStat analysis showing the bacterial abundance at the genus level among the control $(n=4)$, HF $(n=5)$, HF $+S$ $(n=8)$ and HF+SR $(n=8)$ mice (A). Top 35 most frequent bacterial genera in the four different groups (B). Relative abundance of the major phyla among the gut microbiota in the four groups. Bacteroidetes and Firmicutes were the two most abundant bacteria (C). ${ }^{\star} P<0.05$ vs. controls. ${ }^{\star \star} P<0.05$ vs. HF.S group.

infarction (42). However, Marques et al. found that a high fiber diet led to a decrease in the Firmicutes/Bacteroidetes ratio and prevented the development of hypertension and heart failure in hypertensive mice (43). An increased Firmicutes/Bacteroidetes ratio was also observed in hypertensive patients and rats (44). Perhaps the age of the host, the type of disease, the dietary composition, the environment and other factors can all affect the abundance and composition of gut microbiota $(45,46)$. The gut microbiota is significantly associated with some polyamines including spermidine. In addition to synthesizing spermidine (14), some gut microorganisms, including Firmicute species, also contain spermidine synthase and some accumulate spermidine as the sole polyamine (15). Therefore, the levels and function of spermidine and the microbial community richness or diversity can affect each other. Moreover, decreasing spermidine resulted in the deterioration of $\mathrm{HF}$ and a reduction in the Firmicutes/Bacteroidetes ratio. Thus, spermidine may also play beneficial roles by optimizing the gut microbiota composition.
Several limitations should be discussed. First, in this study, the timing of the administration of spermidine or its antagonist was only 1 week, which was shorter than other reports in which spermidine was administered for 612 weeks or longer $(13,14)$. It is possible that a significant beneficial effect of spermidine in improving cardiac function might be seen if it was administered for a longer period of time. Second, to avoid the effects of sex differences on the roles of spermidine, only female mice were used in this study. The roles of spermidine in cardiac function in male mice need to be investigated further. Third, we did not investigate the appropriate concentrations of spermidine for improving cardiac function and did not measure the variation of spermidine in the blood. Last, our results showed that the abundance of Millionella massiliensis decreased significantly in HF mice treated with 4-MCHA, a spermidine synthase inhibitor. However, spermidine can be synthesized by some gut microorganisms. We did not investigate whether Millionella massiliensis possessed much greater spermidine 
synthesis ability than other microbiota and 4-MCHA acted as a spermidine synthase inhibitor by decreasing the abundance of Millionella massiliensis.

In summary, these findings showed that inhibiting spermidine by inhibiting its synthesis deteriorated cardiac function, while increasing spermidine improved cardiac function, and the regulation of gut microbiota and cardiac fibrosis might be a factor in the effects of spermidine on the modulation of cardiac function.

\section{DATA AVAILABILITY STATEMENT}

The original contributions presented in the study are included in the article/Supplementary Material, further inquiries can be directed to the corresponding author.

\section{ETHICS STATEMENT}

The animal study was reviewed and approved by Institutional Ethics Committee of Guangzhou First People's Hospital.

\section{REFERENCES}

1. Sandek A, Bjarnason I, Volk HD, Crane R, Meddings JB, Niebauer J, et al. Studies on bacterial endotoxin and intestinal absorption function in patients with chronic heart failure. Int J Cardiol. (2012) 157:805. doi: 10.1016/j.ijcard.2010.12.016

2. Morris NL, Hammer AM, Cannon AR, Gagnon RC, Li X, Choudhry MA. Dysregulation of microRNA biogenesis in the small intestine after ethanol and burn injury. Biochim Biophys Acta Mol Basis Dis. (2017) 1863:264553. doi: 10.1016/j.bbadis.2017.03.025

3. Sandek A, Swidsinski A, Schroedl W, Watson A, Valentova M, Herrmann R, et al. Intestinal blood flow in patients with chronic heart failure: a link with bacterial growth, gastrointestinal symptoms, and cachexia. J Am Coll Cardiol. (2014) 64:1092-102. doi: 10.1016/j.jacc.2014.06.1179

4. Kummen M, Mayerhofer CCK, Vestad B, Broch K, Awoyemi A, StormLarsen C, et al. Gut microbiota signature in heart failure defined from profiling of 2 independent cohorts. J Am Coll Cardiol. (2018) 71:118486. doi: 10.1016/j.jacc.2017.12.057

5. Luedde M, Winkler T, Heinsen FA, Ruhlemann MC, Spehlmann ME, Bajrovic A, et al. Heart failure is associated with depletion of core intestinal microbiota. Esc Heart Fail. (2017) 4:282-90. doi: 10.1002/ehf2.12155

6. Tang WH, Wang Z, Levison BS, Koeth RA, Britt EB, Fu X, et al. Intestinal microbial metabolism of phosphatidylcholine and cardiovascular risk. $N$ Engl J Med. (2013) 368:1575-84. doi: 10.1056/NEJMoa1109400

7. Wang Z, Klipfell E, Bennett BJ, Koeth R, Levison BS, Dugar B, et al. Gut flora metabolism of phosphatidylcholine promotes cardiovascular disease. Nature. (2011) 472:57-63. doi: 10.1038/nature09922

8. Li W, Huang A, Zhu H, Liu X, Huang X, Huang Y, et al. Gut microbiotaderived trimethylamine $\mathrm{N}$-oxide is associated with poor prognosis in patients with heart failure. Med J Aust. (2020) 213:374-9. doi: 10.5694/mja2.50781

9. Soda K. The mechanisms by which polyamines accelerate tumor spread. J Exp Clin Cancer Res. (2011) 30:95. doi: 10.1186/1756-9966-30-95

10. Madeo F, Bauer MA, Carmona-Gutierrez D, Kroemer G. Spermidine: a physiological autophagy inducer acting as an anti-aging vitamin in humans? Autophagy. (2019) 15:165-68. doi: 10.1080/15548627.2018.15 30929

11. Minois N, Carmona-Gutierrez D, Madeo F. Polyamines in aging and disease. Aging (Albany NY). (2011) 3:716-32. doi: 10.18632/aging. 100361

\section{AUTHOR CONTRIBUTIONS}

PC designed the study. Material preparation and data collection were performed by ZG, YC, ZL, and SL. ZG and YC conducted the statistical analyses. PC, ZG, and YC drafted the paper, which was reviewed by all authors. All authors read and approved the final manuscript.

\section{FUNDING}

This work was supported by Natural Science Foundation of Guangdong Province (Grant Number 2020A1515010384), the Science and Technology Planning Project Foundation of Guangzhou (Grant Number 201804010463), and National Natural Science Foundation of China (Grant Number 81770398).

\section{SUPPLEMENTARY MATERIAL}

The Supplementary Material for this article can be found online at: https://www.frontiersin.org/articles/10.3389/fcvm. 2021.765591/full\#supplementary-material

12. de Cabo R, Carmona-Gutierrez D, Bernier M, Hall MN, Madeo F. The search for antiaging interventions: from elixirs to fasting regimens. Cell. (2014) 157:1515-26. doi: 10.1016/j.cell.2014.05.031

13. Kiechl S, Pechlaner R, Willeit P, Notdurfter M, Paulweber B, Willeit $\mathrm{K}$, et al. Higher spermidine intake is linked to lower mortality: a prospective population-based study. Am J Clin Nutr. (2018) 108:37180. doi: 10.1093/ajcn/nqy102

14. Eisenberg T, Abdellatif M, Schroeder S, Primessnig U, Stekovic S, Pendl $\mathrm{T}$, et al. Cardioprotection and lifespan extension by the natural polyamine spermidine. Nat Med. (2016) 22:1428-38. doi: 10.1038/nm.4222

15. Yan J, Yan JY, Wang YX, Ling YN, Song XD, Wang SY, et al. Spermidineenhanced autophagic flux improves cardiac dysfunction following myocardial infarction by targeting the AMPK/mTOR signalling pathway. Br J Pharmacol. (2019) 176:3126-42. doi: 10.1111/bph.14706

16. Noack J, Dongowski G, Hartmann L, Blaut M. The human gut bacteria Bacteroides thetaiotaomicron and Fusobacterium varium produce putrescine and spermidine in cecum of pectin-fed gnotobiotic rats. J Nutr. (2000) 130:1225-31. doi: 10.1093/jn/130.5.1225

17. Hanfrey CC, Pearson BM, Hazeldine S, Lee J, Gaskin DJ, Woster PM, et al. Alternative spermidine biosynthetic route is critical for growth of Campylobacter jejuni and is the dominant polyamine pathway in human gut microbiota. J Biol Chem. (2011) 286:43301-12. doi: 10.1074/jbc.M111.307835

18. Ma L, Ni Y, Wang Z, Tu W, Ni L, Zhuge F, et al. Spermidine improves gut barrier integrity and gut microbiota function in diet-induced obese mice. Gut Microbes. (2020) 12:1-19. doi: 10.1080/19490976.2020.1832857

19. Date MO, Morita T, Yamashita N, Nishida K, Yamaguchi O, Higuchi Y, et al. The antioxidant N-2-mercaptopropionyl glycine attenuates left ventricular hypertrophy in in vivo murine pressure-overload model. J Am Coll Cardiol. (2002) 39:907-12. doi: 10.1016/S0735-1097(01)01826-5

20. Chai N, Zhang H, Li L, Yu X, Liu Y, Lin Y, et al. Spermidine prevents heart injury in neonatal rats exposed to intrauterine hypoxia by inhibiting oxidative stress and mitochondrial fragmentation. Oxid Med Cell Longev. (2019) 2019:5406468. doi: 10.1155/2019/5406468

21. Kobayashi M, Watanabe T, Xu YJ, Tatemori M, Goda H, Niitsu M, et al. Control of spermidine and spermine levels in rat tissues by trans-4methylcyclohexylamine, a spermidine-synthase inhibitor. Biol Pharm Bull. (2005) 28:569-73. doi: 10.1248/bpb.28.569

22. Gao XM, Dart AM, Dewar E, Jennings G, Du XJ. Serial echocardiographic assessment of left ventricular dimensions and 
function after myocardial infarction in mice. Cardiovasc Res. (2000) 45:330-8. doi: 10.1016/S0008-6363(99)00274-6

23. Madeo F, Eisenberg T, Pietrocola F, Kroemer G. Spermidine in health and disease. Science. (2018) 359:eaan2788. doi: 10.1126/science.aan2788

24. Ho JE, Liu C, Lyass A, Courchesne P, Pencina MJ, Vasan RS, et al. Galectin-3, a marker of cardiac fibrosis, predicts incident heart failure in the community. J Am Coll Cardiol. (2012) 60:1249-56. doi: 10.1016/j.jacc.2012.04.053

25. Wu J, Zheng H, Liu X, Chen P, Zhang Y, Luo J, et al. Prognostic value of secreted frizzled-related protein 5 in heart failure patients with and without type 2 diabetes mellitus. Circ Heart Fail. (2020) 13:e007054. doi: 10.1161/CIRCHEARTFAILURE.120.007054

26. Yang S, Chen H, Tan K, Cai F, Du Y, Lv W, et al. Secreted frizzled-related protein 2 and extracellular volume fraction in patients with heart failure. Oxid Med Cell Longev. (2020) 2020:2563508. doi: 10.1155/2020/2563508

27. LaRocca TJ, Gioscia-Ryan RA, Hearon CM, Jr., Seals DR. The autophagy enhancer spermidine reverses arterial aging. Mech Ageing Dev. (2013) 134:314-20. doi: 10.1016/j.mad.2013.04.004

28. Michiels CF, Kurdi A, Timmermans JP, De Meyer GRY, Martinet W. Spermidine reduces lipid accumulation and necrotic core formation in atherosclerotic plaques via induction of autophagy. Atherosclerosis. (2016) 251:319-27. doi: 10.1016/j.atherosclerosis.2016.07.899

29. de la Pena NC, Sosa-Melgarejo JA, Ramos RR, Mendez JD. Inhibition of platelet aggregation by putrescine, spermidine, and spermine in hypercholesterolemic rabbits. Arch Med Res. (2000) 31:546-50. doi: 10.1016/S0188-4409(00)00238-1

30. Yadav M, Parle M, Jindal DK, Sharma N. Potential effect of spermidine on GABA, dopamine, acetylcholinesterase, oxidative stress and proinflammatory cytokines to diminish ketamine-induced psychotic symptoms in rats. Biomed Pharmacother. (2018) 98:207-13. doi: 10.1016/j.biopha.2017.12.016

31. Del Rio B, Redruello B, Linares DM, Ladero V, Ruas-Madiedo P, Fernandez M, et al. Spermine and spermidine are cytotoxic towards intestinal cell cultures, but are they a health hazard at concentrations found in foods? Food Chem. (2018) 269:321-26. doi: 10.1016/j.foodchem.2018.06.148

32. Weiss TS, Bernhardt G, Buschauer A, Thasler WE, Dolgner D, Zirngibl $\mathrm{H}$, et al. Polyamine levels of human colorectal adenocarcinomas are correlated with tumor stage and grade. Int J Colorectal Dis. (2002) 17:3817. doi: $10.1007 / \mathrm{s} 00384-002-0394-7$

33. Wang CH, Cheng ML, Liu MH, Kuo LT, Shiao MS. Metabolic profile provides prognostic value better than galectin-3 in patients with heart failure. J Cardiol. (2017) 70:92-8. doi: 10.1016/j.jjcc.2016.10.005

34. Chen J, Yaniz-Galende E, Kagan HJ, Liang L, Hekmaty S, Giannarelli C, et al. Abnormalities of capillary microarchitecture in a rat model of coronary ischemic congestive heart failure. Am J Physiol Heart Circ Physiol. (2015) 308:H830-40. doi: 10.1152/ajpheart.00583.2014

35. Cai X, Liu X, Sun L, He Y, Zheng S, Zhang Y, et al. Prediabetes and the risk of heart failure: a meta-analysis. Diabetes Obes Metab. (2021) 23:174653. doi: $10.1111 /$ dom. 14388

36. Zheng H, Zhu H, Liu X, Huang X, Huang A, Huang Y. Mitophagy in diabetic cardiomyopathy: roles and mechanisms. Front Cell Dev Biol. (2021) 9:750382. doi: $10.3389 /$ fcell.2021.750382

37. Wierich MC, Schipke J, Brandenberger C, Abdellatif M, Eisenberg T, Madeo F, et al. Cardioprotection by spermidine does not depend on structural characteristics of the myocardial microcirculation in aged mice. Exp Gerontol. (2019) 119:82-8. doi: 10.1016/j.exger.2019.01.026
38. Clavel T, Lagkouvardos I, Blaut M, Stecher B. The mouse gut microbiome revisited: from complex diversity to model ecosystems. Int J Med Microbiol. (2016) 306:316-27. doi: 10.1016/j.ijmm.2016.03.002

39. Mariat D, Firmesse O, Levenez F, Guimaraes V, Sokol H, Dore J, et al. The Firmicutes/Bacteroidetes ratio of the human microbiota changes with age. BMC Microbiol. (2009) 9:123. doi: 10.1186/1471-2180-9-123

40. Sun L, Jia H, Li J, Yu M, Yang Y, Tian D, et al. Cecal gut microbiota and metabolites might contribute to the severity of acute myocardial ischemia by impacting the intestinal permeability, oxidative stress, and energy metabolism. Front Microbiol. (2019) 10:1745. doi: 10.3389/fmicb.2019. 01745

41. Lucking EF, O'Connor KM, Strain CR, Fouhy F, Bastiaanssen TFS, Burns DP, et al. Chronic intermittent hypoxia disrupts cardiorespiratory homeostasis and gut microbiota composition in adult male guinea-pigs. Ebiomedicine. (2018) 38:191-205. doi: 10.1016/j.ebiom.2018.11.010

42. Lataro RM, Imori PFM, Santos ES, Silva LEV, Duarte RTD, Silva CAA, et al. Heart failure developed after myocardial infarction does not affect gut microbiota composition in the rat. Am J Physiol Gastrointest Liver Physiol. (2019) 317:G342-G48. doi: 10.1152/ajpgi.00018.2019

43. Marques FZ, Nelson E, Chu PY, Horlock D, Fiedler A, Ziemann $M$, et al. High-fiber diet and acetate supplementation change the gut microbiota and prevent the development of hypertension and heart failure in hypertensive mice. Circulation. (2017) 135:964-77. doi: 10.1161/CIRCULATIONAHA.116.024545

44. Yang T, Santisteban MM, Rodriguez V, Li E, Ahmari N, Carvajal JM, et al. Gut dysbiosis is linked to hypertension. Hypertension. (2015) 65:133140. doi: 10.1161/HYPERTENSIONAHA.115.05315

45. Turnbaugh PJ, Hamady M, Yatsunenko T, Cantarel BL, Duncan A, Ley RE, et al. A core gut microbiome in obese and lean twins. Nature. (2009) 457:480-4. doi: 10.1038/nature 07540

46. Hill JE, Hemmingsen SM, Goldade BG, Dumonceaux TJ, Klassen J, Zijlstra RT, et al. Comparison of ileum microflora of pigs fed corn-, wheat-, or barley-based diets by chaperonin-60 sequencing and quantitative PCR. Appl Environ Microbiol. (2005) 71:867-75. doi: 10.1128/AEM.71.2. 867-875.2005

Conflict of Interest: The authors declare that the research was conducted in the absence of any commercial or financial relationships that could be construed as a potential conflict of interest.

Publisher's Note: All claims expressed in this article are solely those of the authors and do not necessarily represent those of their affiliated organizations, or those of the publisher, the editors and the reviewers. Any product that may be evaluated in this article, or claim that may be made by its manufacturer, is not guaranteed or endorsed by the publisher.

Copyright () 2021 Chen, Guo, Li, Liu and Chen. This is an open-access article distributed under the terms of the Creative Commons Attribution License (CC BY). The use, distribution or reproduction in other forums is permitted, provided the original author(s) and the copyright owner(s) are credited and that the original publication in this journal is cited, in accordance with accepted academic practice. No use, distribution or reproduction is permitted which does not comply with these terms. 